\title{
SW-Filtering: An Efficient Traffic Classification Method Based on Convolutional Neural Networks
}

\author{
He Huang, Haojiang Deng, Jun Chen ${ }^{+}$, Zhichuan Guo and Runzi Zhang \\ National Network New Media Engineering Research Center, Institute of Acoustics, University of Chinese \\ Academy of Science, Beijing, 100190, China
}

\begin{abstract}
We propose a compact and effective CNN(convolutional neural network)-based method for real time traffic classification. Firstly, we apply a stochastic strategy in the convolutional pooling filter, so as to prevent overfitting and enhance local features. We implement the CNN architecture with one-dimensional convolutional kernel. Furthermore, we stochastically select flows from the BoFs (bag of flows) to classify in each time period and record each classification result. Finally, by taking all these results into voting, we obtain the final results for each $\mathrm{BoF}$ (all the flows in one $\mathrm{BoF}$ share one label). This method not only prevents some inherent problems of CNN-based classifiers, such as overfitting and patterns down-weighting, but is also efficient in memory consumption. Our mathematical proofs and experiments demonstrated that the proposed method can significantly improve the performance of CNN-based traffic classifiers in both accuracy and space efficiency.
\end{abstract}

Keywords: Deep learning; traffic classification; convolutional neural networks; stochastic window filtering;

\section{Introduction}

For the past few years, deep learning methods have been applicated to solve various classification problems, including network traffic classification [1]. However, in spite of their high accuracy and outperformance in feature extraction, these methods suffer from high overfitting rate [4]. The main reason is that they own large sets of parameters compared to the small size of their target traffic data, which is a critical obstacle to generalize them to other network scenarios.

To cope with the overfitting problem, we apply one-dimensional CNN architecture and a stochastic pooling-filter strategy in the classifier. Our pooling filter regards its elements as a probability distribution, then randomly generates the output according to the distribution. We designed this method in order to get well tradeoff between the global and local features.

To deal with the resource consumption, we adopted the BoF (Bag of traffic Flows) model [7] and a timevarying voting policy. Traditional voting policy calls all the flows in the bag in each individual time period, whereas our policy stochastically chooses part of the flows to operate by period. We maintained a table to record how many time each flow is called, thus preventing repetitive computation. We conducted comparison experiments to ensure this method won't cause a significant drop in classification accuracy.

This paper principally makes the contributions as following: Firstly, we presented a novel stochastic pooling method in the CNN-based traffic classification system to minimize the classification error. Our method is designed according to the spatial characteristics of flow contents, reducing the overfitting rate as

\footnotetext{
+ Corresponding author. Tel.: + 8613683176444

E-mail address:chenj@dsp.ac.cn.
} 
much as possible. Moreover, we adopted a stochastic flow selecting method in the test stage so as to decrease memory consumption.

Although massive studies have been carried out on the application of CNN in traffic classification [2]-[5], the balance of classification abilities and efficiency of the classifier still remains to be explored. Our work tried to enhance the classification by reducing overfitting rate and memory consumption without losing accuracy.

\section{Methodology}

Our method applies a CNN architecture with 2 convolutional layers and 2 pooling filters as the classifier. Firstly, we alternate the pooling layers from common-used max-pooling style into our stochastic style and establish the training. Then we arrange all the testing traffic in the form of BoF and test each of them with the alternated classifier. Next, we apply the Stochastic Voting Window to make the flows in each BoF to vote for their common labels. Finally, we get the classification result of all the testing traffic. The input for $\mathrm{CNN}$ is the first 720 bytes of each flow package. Once the piece is shorter than 720 , we will fill 0x00s in the end to fulfill the input format.

\subsection{Stochastic Pooling Window}

In practical terms, there is no denying that the major feature of a flow type often manifests as some outstanding bytes (such as 0xff) or some fixed orders of bytes. Conventionally, max pooling outperforms mean pooling in this scenario. Max pooling record the largest value in each pooling window (or pooling filter) as the output. This pooling strategy is concise and efficient but tends to cause overfitting on small data. Moreover, once the window size is fixed, the resolution of the CNN is fixed and may be insensitive to some specific kinds of traffic features, which further harms the classifier's extensiveness. As such, we design a stochastic pooling window, based on multinomial distribution, to deeply parse the features of traffic.

Unlike commonly-used one-staged pooling window, our traffic pooling window consists of 2 stages, the

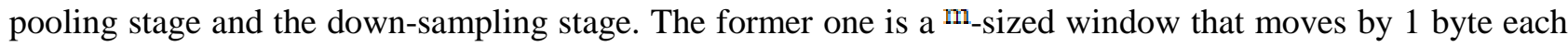
time, refines a byte according to the pooling function and finally returns a sequence as large as the original traffic. The latter one takes the sequence as input, then chooses one in every $\mathrm{m}$ bytes.to construct the output. Consequently, the output's size is one ${ }^{\mathrm{m}}$-ths of the original traffic.

To be more precise, setting $\mathrm{n}$ as the size of the original traffic, $x_{k i}$ as the i-th byte of current windowfilter $F_{k}(k \leq n), \mathrm{m}$ as the size of $F_{k}$, the probability $\mathrm{p}$ of each byte is calculated as equation (1):

$$
p_{k i}=\frac{x_{k i}}{\sum_{x_{j} \in P_{i k}} x_{j}}
$$

Thus, we get the multinomial distribution of $\mathrm{p}:\left\{p_{1}, p_{2}, \ldots x p_{m}\right\}$. The corresponding byte ( pool $\left._{k} k \leq n\right)$ of window-filter $F_{k}$ is stochastically generated according to it, as shown in equation (2):

$$
\begin{gathered}
\operatorname{Pr}\left(\text { pool }_{k}=x_{k 1}\right)=p_{k 1} \\
\vdots \\
\operatorname{Pr}\left(\text { pool }_{k}=x_{k m}\right)=p_{k m}
\end{gathered}
$$

Next, we come to the 2 nd stage. A random number ${ }^{\mathrm{r}}$ between 1 and $\mathrm{m}$ is selected to generate output. The final output is shown in equation (3):

$$
\left\{x_{1 y}, x_{2 y}, x_{2 y, m}\right\}
$$

The complete procedure is described in 0 . The probabilities of pool $_{2}=6$ and ${ }^{\text {oool }_{5}}=7$ are 0.38 and 0.35 respectively, while the probabilities of $\mathrm{pool}_{2}=7$ and pool $_{5}=10$ are 0.38 and 0.50 respectively. If here we use max pooling or mean pooling, then these two values are fixed to be 7 and 10. As such, the classifier is losing the information of training samples' entropy, thus increasing the probability of overfitting. Our method guarantees that those non-extreme values will be considered as well, so as to improve the performance of the classifier. 


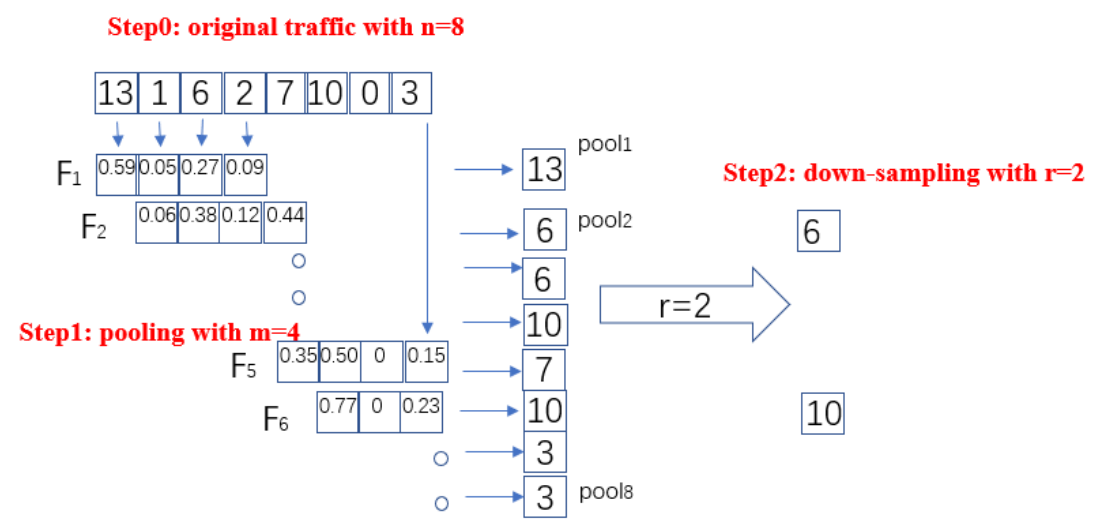

Fig. 1: Simple example for stochastic filter

\subsection{Stochastic Voting Window}

Classifiers based on BoF model are supposed to be primary dynamic programming problems [6], that is to say, they must keep the history state of every flow in memory to compute the result. This strategy can lower the time complexity of state equations and obtain high accuracy, but will make the total time and space consumption relatively high. To cope with these drawbacks, we proposed BoF-based SVW (Stochastic Voting Window) method. SVW is capable of implementing the classification in higher time and space efficiency without losing much accuracy. Since CNN structure can provide high accuracy, a little drop of accuracy in the voting stage is tolerable.

It is easy to understand that, since a BoF is a set of similar flows, the initial classification determines most flows' final labels. The coming iterative process is an alternation on the result. In order to enhance the classification efficiency, we made effort to simplify the alternation stage. We set up a voting value (V-value) for each class in every BoF. In each iteration, once a voter flow is classified into class $i$, the $i$-th $V$-value will increase by 1 . Let $\mathrm{m}$ being the size of $\mathrm{V}$-value set, $\mathrm{n}$ being the number of flows in a BoF, $\mathrm{p}$ being the selection probability, sv being the stop value, $\mathrm{dr}$ as the decaying rate, et as the eliminating threshold, the SVW procedure can be described as below:

i. Train the CNN-based classifier with training sets collected from real-time network environment.

ii. Divide the whole test dataset into several BoFs according to the identical triples (protocol, IP of destination and port of destination).

iii. For each BoF, call the classifier and obtain each individual flow's label. Record the majority's label as the initial label of the BoF.

iv. If the percentage of the majority's vote is larger than sv or if the operational time is big enough, terminate the voting process and set the current label as the final result. Otherwise, go to step v.

v. In the next time period, multiple all the votes on V-value table with dr. If any V-value gets below et, drop it.

vi. Then, select a couple of flows in the majority with probability $\mathrm{p}$ and another couple in the minority. Thus we create a window to filter out some redundant flows. The window size of the 2 couples should be as large as $\frac{1}{2} n$. Call the classifier and obtain the votes of the flows in the window. Renew the V-value table according to the votes and go to step iv.

In this procedure, step i to iii are initializations and the remainder steps are loops to optimize the result. The features and advantages of the remainder steps are discussed as following.

1) Step iv demonstrates the terminal condition of the loop, which will stop the procedure at a proper degree. This method can lower the time complexity.

2) Step $\mathrm{v}$ presents the time-varying rule of $\mathrm{V}$-value. The latest votes will have the strongest influence. Therefore we can consider the time information without increasing time and memory consumption.

3) Notably, according to step vi, classifier only need to deal with a window of $\frac{1}{2} n$ flows at one time, saving much memory usage

\section{Experiments}

In this section, several experiments were conducted on real-time traffics to evaluate our Stochastic Window Filtering methods, SPW mentioned in subsection 2.1 and SVW mentioned in subsection 2.2. The experimental data is listed in Table 1. 
Table 2: Flows captured for experiments

\begin{tabular}{|l|l|l|}
\hline Protocol & Number & Percentage \\
\hline DB2 & 22357 & $11.15 \%$ \\
\hline DNS & 10004 & $4.99 \%$ \\
\hline FTP & 10052 & $5.01 \%$ \\
\hline HTTP & 46893 & $23.37 \%$ \\
\hline MODBUS TCP & 19025 & $9.49 \%$ \\
\hline ORACLE & 18796 & $9.37 \%$ \\
\hline SMTP & 20499 & $10.22 \%$ \\
\hline SSH & 9156 & $4.57 \%$ \\
\hline UNKNOWN & 43781 & $21.83 \%$ \\
\hline TOTAL & 200563 & $100 \%$ \\
\hline
\end{tabular}

Each individual test flow was classified by rare 1D-CNN, 1D-CNN with SPW and 1D-CNN with 2 SWFiltering methods respectively. Therefore, we got 3 series of testing results. The F-measure values of each series were calculated to evaluate the performance of each classifier, which is shown in Fig. 2. The histograms demonstrated that the F-measures of most flows were improved observably with the proposed methods. Particularly, the SSH protocol did not obtain a better performance with SW-Filters. The reason is very likely to be that the shell format of the protocol is relatively simple and fixed, so that our SW-Filtering methods are in vain. Other probable reasons include that the rare 1D-CNN already suffered from overfitting. Our methods performed better on the protocols with distinguished pattern and various parameters, such as DNS and MODBUS, on which the improvements were over 5\%. On contrary, HTTP did not get much improvement due to its relatively complex structure and payloads, on which the improvements were only about 2\%. The raw classification data is displayed in Table 2. and Table 3. According to the tables, the classifier with our method is more likely not to classify the flows into "UNKNOWN" class, which demonstrates that our method can help the classifier to record the flow-patterns more detailedly.

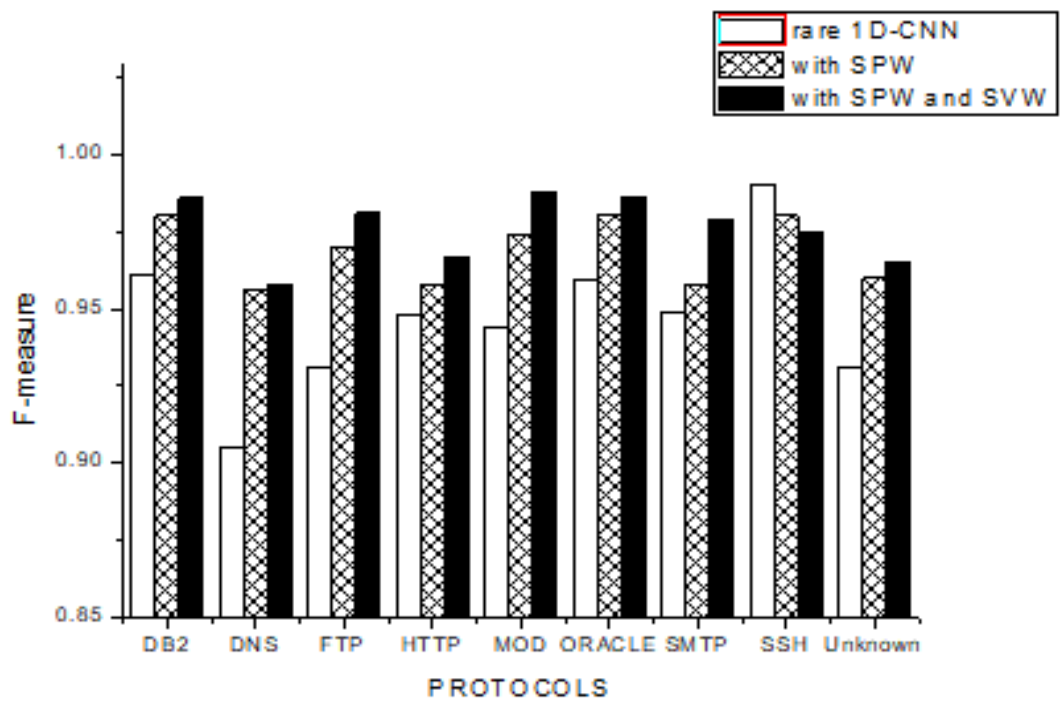

Fig. 2: F-measures for each protocol using different classifiers

Table 2: Performance of 1D-CNN-based classifier without SW-Filtering methods

\begin{tabular}{|c|c|c|c|c|c|c|c|c|c|}
\hline Protocol & DB2 & DNS & FTP & HTTP & MODBUS-TCP & ORACLE & SMTP & SSH & UNKNOWN \\
\hline DB2 & 0.952 & 0 & 0 & 0 & 0 & 0.036 & 0 & 0 & 0.012 \\
\hline DNS & 0 & 0.906 & 0 & 0 & 0.042 & 0 & 0.018 & 0.004 & 0.030 \\
\hline FTP & 0 & 0.022 & 0.898 & 0.018 & 0 & 0 & 0.048 & 0.014 & 0 \\
\hline HTTP & 0 & 0 & 0.032 & 0.942 & 0 & 0 & 0.016 & 0 & 0.010 \\
\hline MODBUS-TCP & 0 & 0.050 & 0 & 0 & 0.932 & 0 & 0 & 0 & 0.018 \\
\hline ORACLE & 0.028 & 0 & 0 & 0 & 0 & 0.954 & 0 & 0 & 0.018 \\
\hline SMTP & 0 & 0 & 0 & 0.010 & 0 & 0 & 0.978 & 0 & 0.012 \\
\hline SSH & 0 & 0 & 0 & 0 & 0 & 0 & 0 & 0.998 & 0.002 \\
\hline UNKNOWN & 0 & 0.024 & 0 & 0.016 & 0 & 0 & 0 & 0 & 0.960 \\
\hline
\end{tabular}


Table 3: Performance of 1D-CNN-based classifier with SW-Filtering methods

\begin{tabular}{|l|l|l|l|l|l|l|l|l|l|}
\hline Protocol & DB2 & DNS & FTP & HTTP & $\begin{array}{l}\text { MODBUS- } \\
\text { TCP }\end{array}$ & ORACLE & SMTP & SSH & UNKNOWN \\
\hline DB2 & 0.988 & 0 & 0 & 0 & 0 & 0.012 & 0 & 0 & 0 \\
\hline DNS & 0 & 0.940 & 0 & 0.008 & 0 & 0 & 0 & 0.040 & 0.012 \\
\hline FTP & 0 & 0.008 & 0.978 & 0 & 0 & 0 & 0.014 & 0 & 0 \\
\hline HTTP & 0 & 0 & 0.016 & 0.956 & 0 & 0 & 0.006 & 0.006 & 0.016 \\
\hline MODBUS-TCP & 0 & 0.024 & 0 & 0 & 0.976 & 0 & 0 & 0 & 0 \\
\hline ORACLE & 0.016 & 0 & 0 & 0 & 0 & 0.984 & 0 & 0 & 0 \\
\hline SMTP & 0 & 0.004 & 0 & 0.012 & 0 & 0 & 0.978 & 0 & 0.006 \\
\hline SSH & 0 & 0 & 0 & 0 & 0 & 0 & 0 & 0.994 & 0.006 \\
\hline UNKNOWN & 0 & 0 & 0 & 0 & 0 & 0 & 0 & 0 & 0.970 \\
\hline
\end{tabular}

\section{Conclusions and Future Work}

In this work, we proposed the SW-Filtering methods for CNN-and-BoF-based traffic classifiers, which can efficiently classify flows according to their application labels. We chose CNN as the classifier's architecture because it can ignore the complicated feature engineering and can obtain high classification accuracy. Preliminary experiments conducted on common real-time flows have proved that our SW-Filtering methods can improve the current classifiers on F-measure obviously. We plan to conduct further experiments to test the filter's ability in saving memory. This method's outstanding performance on DNS, MODBUS and UNKNOWN demonstrates their potential on detecting unknown types of intrusion towards Industrial Control Systems (ICS). This topic is promising since the related application layer protocols tend to be more diverse and multi-functional in the future.

\section{Acknowledgement}

The authors would like to thank the reviewers for their helpful comments and advice. This work has been supported by the First-Action Program of Institute of Acoustics, Chinese Academy of Sciences (SXJH201609).

\section{References}

[1] Buczak, A. L. and Guven, E. (2016). A survey of data mining and machine learning methods for cyber security intrusion detection. IEEE Communications Surveys \& Tutorials, 18(2), 1153-1176.

[2] Kolosnjaji, B., Zarras, A., Webster, G., \& Eckert, C. (2016, December). Deep learning for classification of malware system call sequences. In Australasian Joint Conference on Artificial Intelligence (pp. 137-149). Springer, Cham.

[3] Wang, Q., Guo, W., Zhang, K., Ororbia II, A. G., Xing, X., Liu, X., \& Giles, C. L. (2017, August). Adversary resistant deep neural networks with an application to malware detection. In Proceedings of the 23rd ACM SIGKDD International Conference on Knowledge Discovery and Data Mining (pp. 1145-1153). ACM.

[4] Wu, B., Liu, Z., Yuan, Z., Sun, G., \& Wu, C. (2017, September). Reducing Overfitting in Deep Convolutional Neural Networks Using Redundancy Regularizer. In International Conference on Artificial Neural Networks (pp. 49-55). Springer, Cham.

[5] Yakura, H., Shinozaki, S., Nishimura, R., Oyama, Y., \& Sakuma, J. (2017, November). Malware Analysis of Imaged Binary Samples by Convolutional Neural Network with Attention Mechanism. In Proceedings of the 10th ACM Workshop on Artificial Intelligence and Security (pp. 55-56). ACM.

[6] Zhang, J., Chen, C., Xiang, Y., Zhou, W., \& Xiang, Y. (2013). Internet traffic classification by aggregating correlated naive bayes predictions. IEEE Transactions on Information Forensics and Security, 8(1), 5-15.

[7] Zhang, J., Xiang, Y., Wang, Y., Zhou, W., Xiang, Y., \& Guan, Y. (2013). Network traffic classification using correlation information. IEEE Transactions on Parallel and Distributed systems, 24(1), 104-117. 